\begin{tabular}{|c|c|c|}
\hline \multirow{2}{*}{ IDUNAS } & NATURAL \& APPLIED SCIENCES & 2019 \\
& Vol. 2 \\
No. 2 \\
$(16-29)$
\end{tabular}

\title{
Synthesis and Characterization of Oleic Acid Coated Magnetic Nanoparticles for Hyperthermia Applications \\ Research Article
}

\author{
Fatih Şentürk1* iD, Soner Çakmak2 iD, Göknur Güler Ozturk1 iD \\ IDepartment of Biophysics, Faculty of Medicine, Gazi University, Ankara, Turkey. \\ ${ }_{2}$ Department of Bioengineering, Graduate School of Science and Engineering, Hacettepe University, Ankara, Turkey.
}

\author{
Author E-mail: \\ senturkfatih52@gmail.com \\ *Correspondence to: F. Şentürk, Department of Biophysics, Faculty of Medicine, Gazi University, \\ Ankara, Turkey \\ DOI: 10.38061/idunas.657975
}

Received: December 11, 2019; Accepted: December 31, 2019

\section{Abstract}

The present study focuses on obtaining highly stable iron oxide nanoparticles (IONs), stabilized by the coating of oleic acid (OA) for magnetic nano hyperthermia (MNH) applications. For this purpose, bare and different amounts of oleic acid $(0.2 \%, 0.5 \%$ and $1.0 \%, \mathrm{v} / \mathrm{v})$ coated IONs were prepared by co-precipitation method. Then, their structures, morphologies, magnetic properties and heating abilities were characterized by using suitable techniques. IONs+1.0\%OA nanoparticles showed low agglomeration with high dispersion capacity. Moreover, $1.0 \%$ OA coating showed the highest heating ability with a temperature increase of $\left(25.2{ }^{\circ} \mathrm{C}\right)$ compared to IONs+OA $\left(0.2 \%, 16.4{ }^{\circ} \mathrm{C} ; 0.5 \%, 19{ }^{\circ} \mathrm{C}\right)$, but similar to bare IONs $\left(26.7{ }^{\circ} \mathrm{C}\right)$. The specific absorption rate (SAR) values of bare IONs and IONs+OA $(0.2 \%, 0.5 \%, 1.0 \%$, v/v) were found as $39.50,34.81,23.36$ and $45.98 \mathrm{~W} / \mathrm{g}$, respectively. Our results showed that comparable hyperthermia effect of IONs+1.0\%OA with bare IONs was attributable to their uniform dispersion performance along with higher SAR values. We concluded that the dispersion of hydrophobic IONs+OA in the aqueous medium is one of the critical requirements for increasing temperature in magnetic nano hyperthermia applications.

Keywords: Iron oxide nanoparticles, magnetic hyperthermia, oleic acid, co-precipitation.

\section{INTRODUCTION}

Hyperthermia is introduced as an adjuvant therapy for various cancers, in which the targeted tissue is exposed to high temperatures. Traditional hyperthermia produces the highest temperature gradient on the body's surface that immediately diminishes with distance from the heat-generating sources such as radiofrequency, microwave, laser, ultrasound and magnetic field [1,2]. Therefore, the heat delivery rate to the tumor site remains quite limited. Nanomaterials have the potential for use as the hyperthermia agents that can absorb energy from the external magnetic field and increase the effect of heating in tumor site [3, 4]. 
Magnetic nano hyperthermia $(\mathrm{MNH})$ is a promising therapeutic procedure for the treatment of various cancers. It depends on the idea that magnetic nanoparticles delivered to tumors can generate heat after exposure to the noninvasive magnetic field and consequently induce cell death [5]. The increase of temperatures above $46{ }^{\circ} \mathrm{C}$ lead cancer cells to necrosis, whereas moderate temperature increases up to 41 ${ }^{\circ} \mathrm{C}-45^{\circ} \mathrm{C}$ might change the functionality of intercellular proteins that cause cellular degradation and induce apoptosis $[1,5]$. In contrast to necrosis, hyperthermia induced apoptosis is a preferable cellular death pathway for tumor eradication because it does not cause undesirable effects on healthy tissue $[3,6]$.

Iron oxide nanoparticles (IONs) have received tremendous attention because of their unique magnetic properties, high surface area, magnetothermal effect and biocompatibility. For many years, IONs have been successfully used for bio-applications that include targeted drug delivery, magnetic separation and magnetic nano hyperthermia (MNH) [7-9]. Iron oxide nanoparticles could be utilized as MNH agents to trigger heat-induced cell death [3]. When IONs are targeted or injected into tumor site and exposed to an alternating magnetic field, they become a source of heat which capable of increasing temperature to kill cancer cells [10]. However, the effective interaction between magnetic fields and IONs depends on the physical and chemical properties of nanoparticles, such as size, shape, saturation magnetization, state of aggregation and monodispersibility, etc. [11].

Chemical co-precipitation is the most conventional method for the production of IONs because of its simplicity and productivity. This method is based on the reaction between ferric and ferrous ions in aqueous media under basic conditions. Although gram-scale production and facility are the best-known advantages of the co-precipitation, the obtained IONs are highly agglomerated because of difficulty in controlling particle-particle interactions and distributions [12, 13]. Agglomeration of nanoparticles is a major challenge for biological applications. This also reduces the heating ability of IONs. Therefore, IONs generally coated with surfactants such as oleic acid (OA), polyethylene glycol (PEG), or citric acid. Oleic acid stabilizes the IONs and prevents oxidation or erosion caused by oxygen and acid or base, respectively [14]. Moreover, OA coating facilitates IONs uniform suspension performance such as dispersibility and stability $[12,14]$.

There are some studies investigating OA coating on IONs within low (0-1.5 wt\%) or high (8-96 wt $\%$ ) OA concentration $[15,16]$. Lai et al. investigated low OA concentrations for IONs coating and they only determined optimum OA loading amount in terms of IONs colloidal stability [15]. On the other hand, Ghosh et al. used high OA concentrations and they characterized the heating ability of OA coated IONs [17]. Some other studies functionalized the surface of IONs with using constant OA concentration and the heating potential of the OA coated IONs were determined for hyperthermia applications [18, 19].

Although oleic acid is a commonly used surfactant to encapsulate magnetic nanoparticles, as far as we know, few studies have been carried out on the heating ability of OA coated IONs for hyperthermia studies. Hence, we have investigated the effect of (i) low OA concentrations on physical and chemical properties of iron oxide nanoparticles, (ii) different amounts of nanoparticles and OA concentrations on heating performance of IONs under relatively low alternating magnetic field, with comprehensive characterization techniques. In this study, we have prepared OA coated iron oxide nanoparticles by coprecipitation method. The structure, morphology and magnetic properties of nanoparticles were characterized by using the X-ray diffraction (XRD), Fourier transform infrared spectroscopy (ATR-FTIR), thermogravimetric analysis (TGA), transmission electron microscopy (TEM), dynamic light scattering (DLS) and vibrating sample magnetometer (VSM). Then, the induction heating ability and specific absorption rate (SAR) values of nanoparticles were evaluated under exposure of alternating magnetic field. 
The amount of optimum oleic acid concentration required on the IONs and concentration dependent heating abilities of IONs were investigated for hyperthermia efficiency.

\section{EXPERIMENTAL SECTION}

\subsection{Materials}

Ferric chloride hexahydrate $\left(\mathrm{FeCl}_{3} .6 \mathrm{H}_{2} \mathrm{O}\right)$, ferrous chloride tetrahydrate $\left(\mathrm{FeCl}_{2} .4 \mathrm{H}_{2} \mathrm{O}\right)$ and oleic acid (OA, technical grade, 90\%) were purchased from Sigma Aldrich (USA). Ammonium hydroxide $\left(\mathrm{NH}_{4} \mathrm{OH}\right.$, $25 \%)$ and hydrochloric acid $(\mathrm{HCl}, 37 \%)$ were obtained from Merck (Germany).

\subsection{Synthesis of iron oxide nanoparticles}

Iron oxide nanoparticles were prepared by co-precipitation method according to the Massart's procedure [20]. Ultra-pure water used for solution preparations was degassed with nitrogen for $30 \mathrm{~min}$ before synthesis. In a typical synthesis, aqueous solutions of ferric chloride hexahydrate $(8 \mathrm{~mL} ; 2.162 \mathrm{~g} ; 1$ M) and ferrous chloride tetrahydrate $(2 \mathrm{~mL} ; 0.793 \mathrm{~g} ; 2 \mathrm{M} \mathrm{HCl})$ was added to $100 \mathrm{~mL}$ of ultra-pure water in a three necked flask. Then, the solution temperature was increased step by step to $50{ }^{\circ} \mathrm{C}$ under constant stirring, followed by dropwise addition of ammonium hydroxide $(5.3 \mathrm{~mL} ; 0.7 \mathrm{M})$ solution. After that, the reaction was cooled to room temperature and the resultant suspension was washed three times with ultrapure water to remove excess ammonia. The mixture was located on a neodymium magnet followed by removal of the upper supernatant. Finally, the black precipitate was dried under vacuum for further analysis.

\subsection{Synthesis of oleic acid coated iron oxide nanoparticles}

In situ oleic acid coating of IONs with different concentrations $(0.2 \%, 0.5 \%$ and $1.0 \%$, v/v) were carried out with the same procedure described above where OA was poured into the reaction mixture before the addition of ammonium hydroxide solution. Then, the same as bare IONs synthesis, the reaction was cooled to room temperature and oleic acid coated IONs were washed repeatedly with ultra-pure water and ethanol, respectively. OA coated IONs were collected by magnetic decantation and dried under vacuum for further characterizations. After this point, bare nanoparticles and IONs coated with $0.2,0.5$ and $1.0 \%$, v/v $\mathrm{OA}$ concentrations are abbreviated as IONs, IONs+0.2\%OA, IONs+0.5\%OA and IONs+1.0\%OA, respectively.

\subsection{Characterization of magnetic nanoparticles}

The crystal structure of the iron oxide nanoparticles was investigated by X-ray diffraction analysis (Rigaku, D/Max-B, Tokyo, Japan) using CuK $\alpha$ radiation at a scanning rate of $0.2^{\circ} / \mathrm{min}$, from 20 to $80^{\circ}$. The average crystallite size was calculated based on the XRD pattern according to the Scherrer's equation (1) [21]:

$$
d=0.9 \lambda / \beta \cos \theta
$$


where $d$ is the crystallite size, $\lambda$ is the $\mathrm{X}$-ray wavelength $(\lambda=1.5405 \AA), \beta$ is the full width at half maximum (fwhm) and $\theta$ is Bragg angle.

The presence of various functional groups on the surface of the iron oxide nanoparticles before and after OA coating was analyzed by Attenuated total reflection Fourier Transform Infrared (ATR-FTIR) Spectroscopy (Nicolet iS10, Thermo Scientific, USA) in the range of $1000-4000 \mathrm{~cm}-1$.

The amount of OA coatings was determined by thermogravimetric analysis (TGA) using Exstar 6000 TG/DTA 6300 (SII Nano Technology Inc., Japan). Samples were heated from 30 to $900{ }^{\circ} \mathrm{C}$ with a heating rate $10{ }^{\circ} \mathrm{C} / \mathrm{min}$ under nitrogen atmosphere. Mass losses were quantified from the obtained thermograms.

The morphology and size of the synthesized nanoparticles were examined by using transmission electron microscopy (TEM, FEI Tecnai $\mathrm{G}_{2}$ Spirit BioTwin, USA). The aqueous dispersions of iron oxide nanoparticles were dropped onto copper grid and the samples were dried prior to the analysis. The average sizes and size distributions of iron oxide nanoparticles were calculated from TEM images using ImageJ software (National Institute of Health, USA).

The hydrodynamic size and size distribution of the iron oxide nanoparticles were determined by dynamic light scattering (DLS) experiments using a Zetasizer Nano ZS (Malvern, UK) at room temperature.

The magnetic field dependent magnetization of iron oxide nanoparticles was measured with quantum design physical property measurement system (QD-PPMS, USA) at room temperature in the field range between $-2 T$ and $+2 T$.

\subsection{Heating potential evaluation of iron oxide nanoparticles}

Magnetic hyperthermia experiments were carried out using Easy Heat 8310 system (Ambrell, UK) with a $3.43 \mathrm{~cm}$ diameter ( 8 turns) heating coil. Cold water circulation is provided to keep the coil at ambient temperature. Bare and OA coated IONs $(1-10 \mathrm{mg} / \mathrm{mL})$ were suspended in $2.5 \mathrm{~mL}$ of distilled water in a glass vial and sonicated for 1-2 min to achieve homogeneous dispersions. Then, samples were placed at the center of the coil and induction heating was applied at $100 \mathrm{~A}, 312 \mathrm{kHz}$ frequency during $10 \mathrm{~min}$. Magnetic field strength $(\mathrm{H})$ was calculated from the equation (2) given below [17]:

$$
H=1.257 n i / L(O e)
$$

where $n$ is the number of turns, $i$ is the applied current and $L$ is the diameter of coil in centimeters. Calculated values of magnetic field strength $(\mathrm{H})$ was $295.5 \mathrm{Oe}$ (equivalent to $23.51 \mathrm{kA} / \mathrm{m}$ ). Temperature was measured with fiber optic temperature sensor (Neoptix, Canada).

The efficiency of the heating potential of the magnetic nanoparticles was represented by the specific absorption rate (SAR) or specific loss power (SLP), which is defined as the heating power generated per unit mass $[4,22]$. The magnitude of the SAR was calculated by the equation (3) [23]:

$$
S A R(W / g)=C \frac{m_{\text {sol }}}{m_{\text {mag }}} \frac{\Delta T}{\Delta t}
$$

where the $C$ is specific heat capacity for water $\left(C=4.18 \mathrm{~J} / \mathrm{g} \cdot{ }^{\circ} \mathrm{C}\right), m_{s o l}$ is the mass of entire sample, $m_{m a g}$ is the mass of IONs and $\Delta \mathrm{T} / \Delta \mathrm{t}$ is the initial slope of the time-dependent temperature curve (from 0 to $30 \mathrm{~s}$ ). 


\section{RESULTS AND DISCUSSION}

\subsection{X-ray diffraction}

XRD patterns of bare and OA coated IONs were shown in Fig. 1. The lattice planes represented by the diffraction peak at angles of $30.36^{\circ}, 35.60^{\circ}, 43.51^{\circ}, 57.29^{\circ}$ and $62.96^{\circ}$, respectively, (220), (311), (400), (511) and (440); the planes are consistent with $\mathrm{Fe}_{3} \mathrm{O}_{4}$ pattern with a cubic structure (JCPDS card no. 01075-0449). The position and intensity of all peaks match well with standard $\mathrm{Fe}_{3} \mathrm{O}_{4}$ diffraction [24, 25]. As expected, the diffraction peaks of IONs disturbed and peak broadening was observed as the oleic acid concentration increases (Fig. 1b-d). This pattern was the most apparent on OA concentration of $1.0 \% \mathrm{v} / \mathrm{v}$ (Fig. 1d). In addition, the relative peak intensities of OA coated IONs with different concentrations was lower than bare IONs. Even though the peaks were weakened due to formation of oleic acid layer on the surface of IONs, but their relating lattice planes were still represented. This suggests that oleic acid coating did not much affect the crystal structure of the iron oxide nanoparticles. In addition, XRD also provide an estimate of average crystallite sizes based on Scherrer's equation [21]. The XRD graph was fitted with multiple Gaussian peaks and most intense peak (311) was used to calculate the average crystallite size of the nanoparticles. The average crystallite sizes for bare IONs and OA coated IONs with different concentrations of $0.2,0.5$ and $1.0 \% \mathrm{v} / \mathrm{v}$ were calculated as 8.30, 9.30, 10.40 and $11.10 \mathrm{~nm}$, respectively. OA coating slightly increased the average crystallite size of IONs. Petcharoen et al., reported that the crystallite size of IONs decreased from $16.8 \mathrm{~nm}$ to $13.9 \mathrm{~nm}$ after coating of IONs with OA concentration of $0.8 \%, \mathrm{v} / \mathrm{v}$ [21]. Wang et al. synthesized IONs with higher OA concentrations than used in this study and they also showed size decrease from $19.8 \mathrm{~nm}$ to $15.3 \mathrm{~nm}$ [24]. However, Soares et al. denoted that OA coating did not change the average diameter and crystalline structure of IONs [26]. Also, Wulandari et al.,2019 showed that amount of OA elevated from 1.0 to $1.5 \mathrm{~mL}$, the crystal size slightly increased from 4.73 to $5.69 \mathrm{~nm}$ after coating IONs [27].

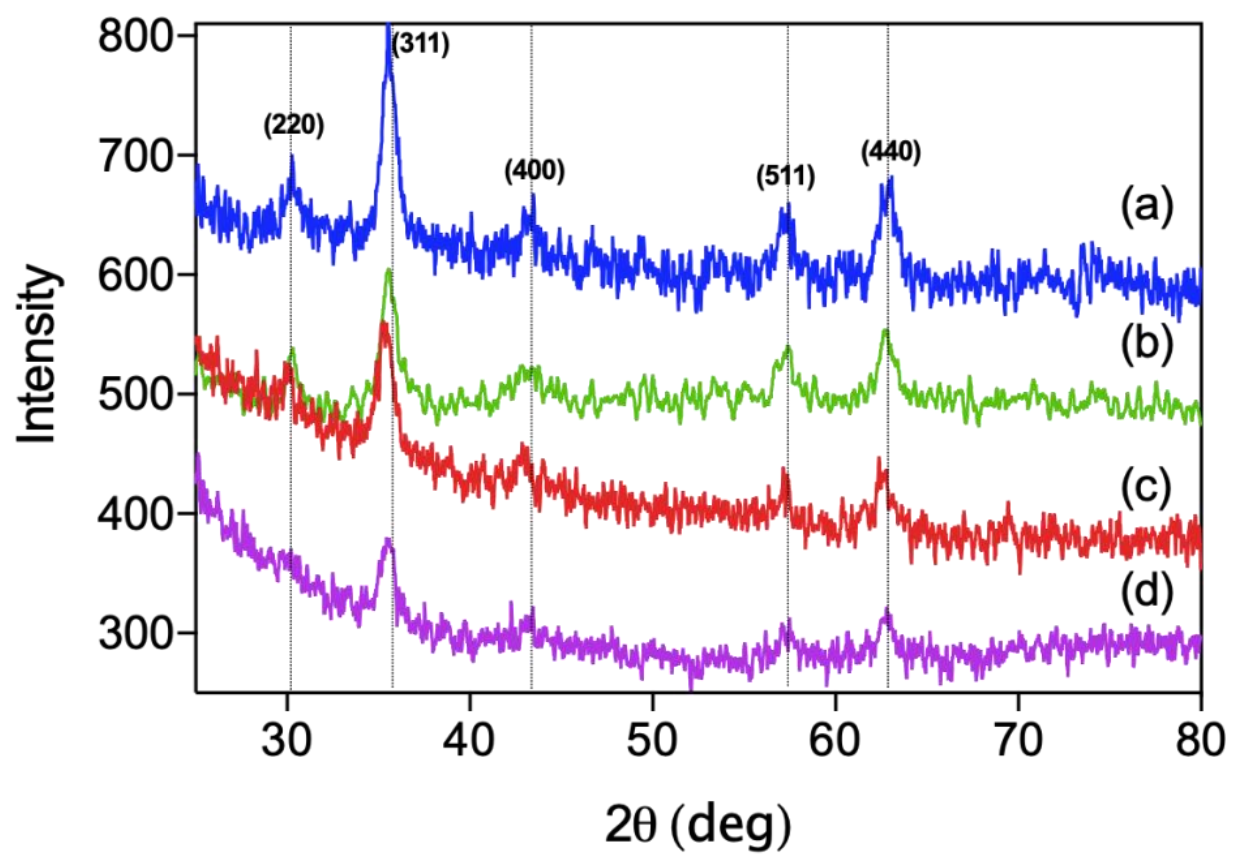

Figure 1. XRD patterns of a) IONs, b) IONs+0.2\%OA, v/v c) IONs+0.5\%OA, v/v and d) IONs+1.0\%OA, $\mathrm{v} / \mathrm{v}$. 


\subsection{Infrared Spectroscopy (ATR-FTIR) analysis}

The adsorption of oleic acid on the surface of IONs was determined by using ATR-FTIR spectroscopy. Fig. 2 shows the ATR-FTIR spectra of OA, bare IONs and OA coated IONs. As can be seen from Fig. 2a that pure OA shows two sharp bands at 2920 and $2855 \mathrm{~cm}-1$ correspond to asymmetric and symmetric $\mathrm{CH}_{2}$ stretchings, respectively. A sharp band seen at $1707 \mathrm{~cm}-1$ corresponds to the stretching vibration of $\mathrm{C}=\mathrm{O}$ and the band at $1286 \mathrm{~cm}-1$ exhibited the presence of the $\mathrm{C}-\mathrm{O}$ stretch present in OA [2831]. Fig. 2b-d reveals the ATR-FTIR spectra obtained from the IONs coated with different concentration $(0.2,0.5,1.0 \%, \mathrm{v} / \mathrm{v})$ of OA. In a co-precipitation method, the surface of the IONs was covered by hydroxyl groups due to the synthesis accomplished in aqueous environment. Thus, the characteristic bands of the hydroxyl groups at 3357 and $1641 \mathrm{~cm}-1$ were observed in the bare IONs (Fig. 2e). After OA coating, the displacement of asymmetric and symmetric $\mathrm{CH}_{2}$ stretching peaks shifted to lower frequencies (2919 and $2852 \mathrm{~cm}-1$ ) for all coatings indicating the OA molecules adsorbed onto the surface of IONs (Fig. 2b-d). This means that the hydrocarbon chains surrounding the IONs were in a close-packed [29, 31-33]. The intense peak at $1707 \mathrm{~cm}-1$ of pure oleic acid disappears in OA coated IONs and instead, the new peaks appear at 1523 and $1404 \mathrm{~cm}-1$ correspond to asymmetric and symmetric vibrations of carboxyl group attached to Fe3+ ions $[28,29]$.

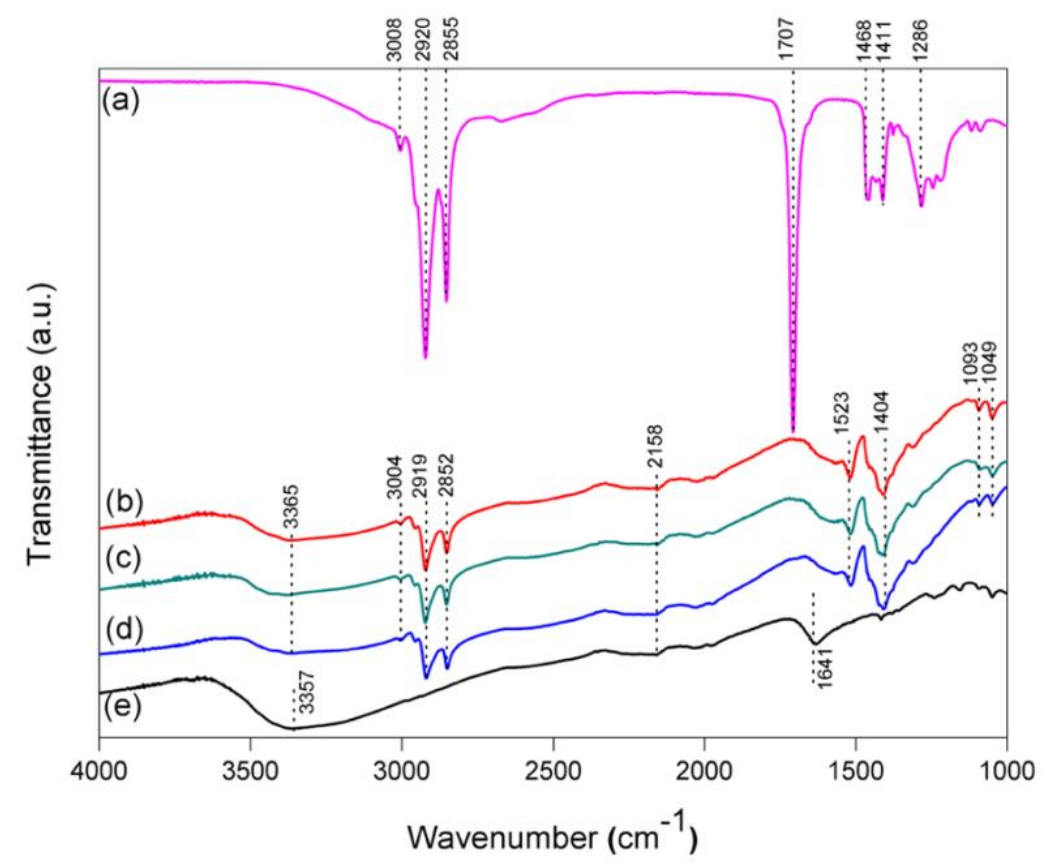

Figure 2. ATR-FTIR spectra of (a) oleic acid. (b) IONs+0.2\%OA, v/v. (c) IONs+0.5\%OA, v/v. (d) IONs+1.0\%OA, v/v. (e) IONs.

\subsection{Thermogravimetric analysis (TGA)}

In order to quantify the amount of $\mathrm{OA}$ associated with IONs, thermogravimetric analysis was performed for bare and OA $\left(0.2 \%, 0.5 \%, 1.0 \%\right.$, v/v) coated IONs from room temperature to $900{ }^{\circ} \mathrm{C}$ (Fig. $3)$. In general, an initial slight weight loss was reported due to evaporation of adsorbed water $[21,34]$. For bare and OA coated IONs, the initial slight weight loss (1-2.5\%) at temperature below $150{ }^{\circ} \mathrm{C}$ refers to the removal of moistures. The weight loss in the temperature range between $200-330{ }^{\circ} \mathrm{C}$ was attributed to removal of organic moieties from the surface of IONs as reported $[35,36]$. Bare IONs showed exhibited 
weight loss $(2.09 \%)$ observed in the temperature range $\sim 200-400{ }^{\circ} \mathrm{C}$ and this may be attributed to the removal of organic groups on the surface of IONs. It is reported in the literature that OA coated IONs show two prominent weight loss steps below $600^{\circ} \mathrm{C}[21,32]$. Also, Mahdavi et al. showed four derivative mass loses in TGA curve, fourth weight loss above $742{ }^{\circ} \mathrm{C}$ is possibly due to deoxidation of FeO [37]. In our study, thermograms of different amounts of oleic acid exhibited three step weight loss. The first step (150$\left.300{ }^{\circ} \mathrm{C}\right)$ is attributed to the free or loosely bound OA, while the second step $\left(300-500{ }^{\circ} \mathrm{C}\right)$ may be due to OA chemically bound to IONs. The third weight loss between 500 and $750{ }^{\circ} \mathrm{C}$ was attributed to the oleic acid-coated layer. Our results (Table 1) showed that the bound OA fraction increased from 2.38 to $4.35 \%$ when the amount of OA used for capping increased from 0.2 to $1.0 \%, \mathrm{v} / \mathrm{v}$.

Table 1. Results and analysis based on TGA curves (Fig. 3) of IONs.

\begin{tabular}{|l|l|l|l|l|l|}
\hline NPs & $\begin{array}{l}\text { Initial weight loss } \\
(\%) \\
\left(0-150{ }^{\circ} \mathrm{C}\right)\end{array}$ & $\begin{array}{l}\text { First weight loss } \\
(\%) \\
\left(150-300{ }^{\circ} \mathrm{C}\right)\end{array}$ & $\begin{array}{l}\text { Second weight } \\
\text { loss }(\%) \\
\left(300-500{ }^{\circ} \mathrm{C}\right)\end{array}$ & $\begin{array}{l}\text { Third weight loss } \\
(\%) \\
\left(500-750{ }^{\circ} \mathrm{C}\right)\end{array}$ & $\begin{array}{l}\text { Total } \\
\text { Weight loss } \\
(\%)\end{array}$ \\
\hline IONs (bare) & 2.50 & $2.09\left(200-400{ }^{\circ} \mathrm{C}\right)$ & ---- & --- & 4.59 \\
\hline IONs+0.2\%OA & 1.15 & 3.95 & 2.38 & 8.9 & 16.38 \\
\hline IONs+0.5\%OA & 2.50 & 3.64 & 3.36 & 8.32 & 17.82 \\
\hline IONs+1.0\%OA & 1.58 & 3.86 & 4.35 & 9.0 & 18.79 \\
\hline
\end{tabular}

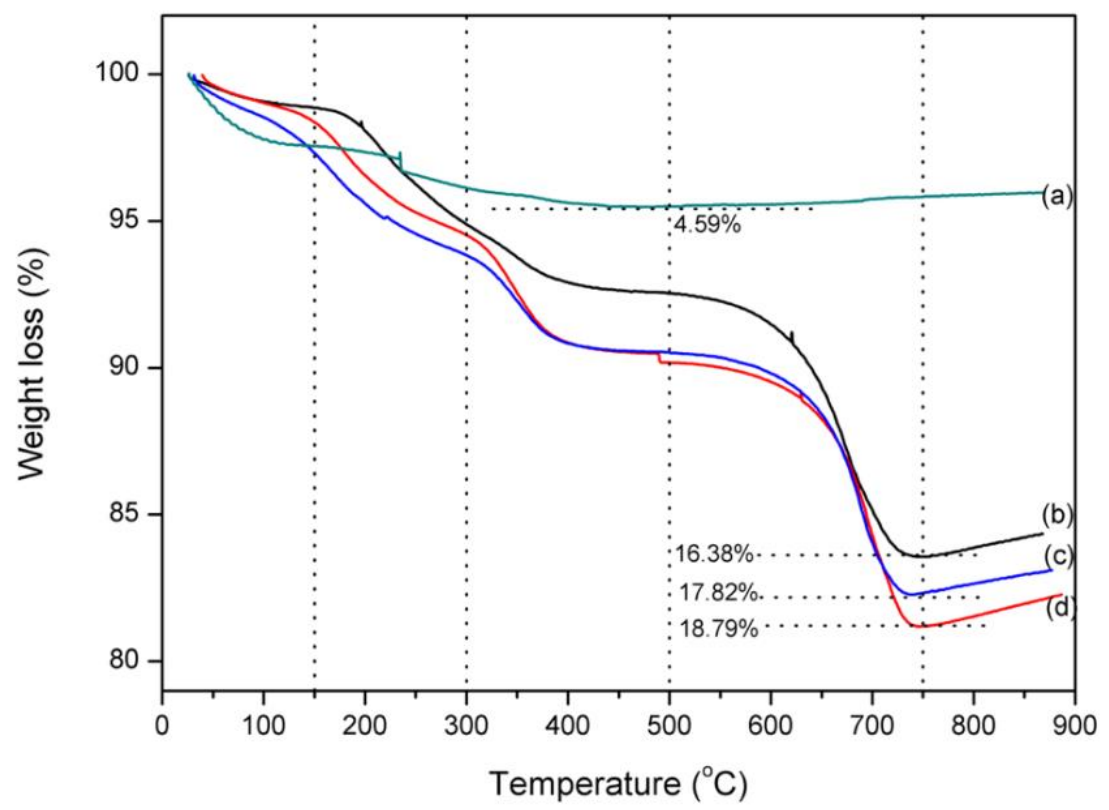

Figure 3. TGA curves of a) IONs b) IONs+0.2\%OA, c) IONs+0.5\%OA, d) IONs+1.0\%OA.

\subsection{Transmission electron microscopy (TEM) analysis}

The size and the morphology of IONs were assessed by using transmission electron microscopy. TEM image of the bare IONs have a narrow size distribution with an average diameter of $8.0 \pm 1.9 \mathrm{~nm}$ (Fig. 4a); however, the particles tend to agglomerate. On the other hand, the IONs prepared with $0.2,0.5$ and $1.0 \% \mathrm{v} / \mathrm{v}$ OA concentrations have the average diameters of $9.3 \pm 2.0 \mathrm{~nm}, 8.3 \pm 1.6 \mathrm{~nm}$ and $10.0 \pm 2.0 \mathrm{~nm}$, respectively. These results showed that OA coatings did not dramatically change the average diameters of IONs and their values were in good agreement with XRD size analyses. The morphology of the bare IONs showed irregular shape (Fig. 4a), on the other hand, IONs coated with OA changed their morphology to 
spherical shape as the OA concentration increased. Moreover, IONs especially prepared in the presence of $1.0 \% \mathrm{v} / \mathrm{v}$ OA were separated from its adjacent particles and adsorbed surfactant on the surface reduced agglomeration. These results suggested that IONs can be homogeneously coated with $1.0 \%$, v/v OA and colloidal stability of IONs were improved with the OA concentration increased from 0.2 to $1.0 \%$, v/v. Petcharoen et al. showed that the OA coating agents provided larger particle sizes due to the combination of the OA layer on the surface of magnetite. According to their SEM (scattering electron microscope) image, bare and OA $(0.8 \%, \mathrm{v} / \mathrm{v})$ coated magnetite nanoparticles have 15 and $27 \mathrm{~nm}$ average diameter, respectively [21]. Unlike the previous study, Madhavi et al. demonstrated that bare and $\mathrm{OA}\left(1.7 \%\right.$, v/v) coated $\mathrm{Fe}_{3} \mathrm{O}_{4}$ NPs sizes were 16.5 and $7.3 \mathrm{~nm}$, respectively. And, bare $\mathrm{Fe}_{3} \mathrm{O}_{4} \mathrm{NPs}$ were seriously aggregated according to TEM image [37]. It is notable that, there are many studies with different results about the effect of coating OA on the nanoparticle size.

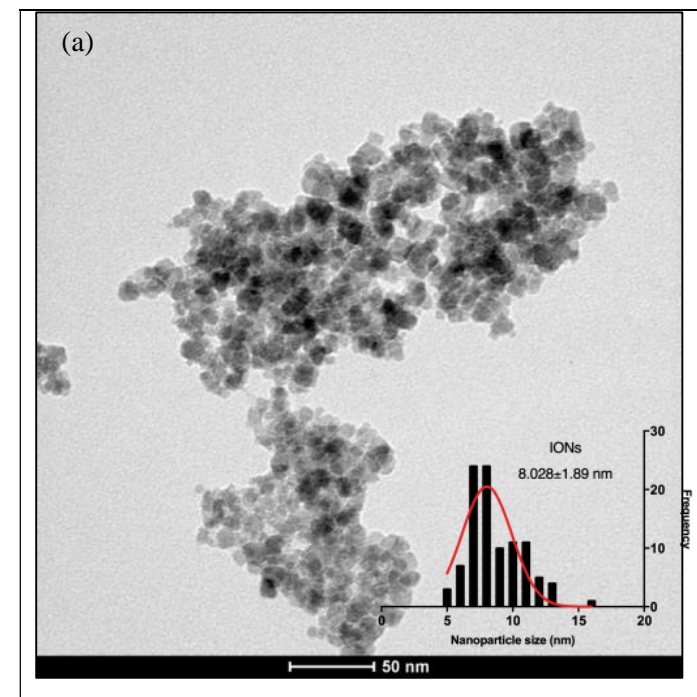

(c)

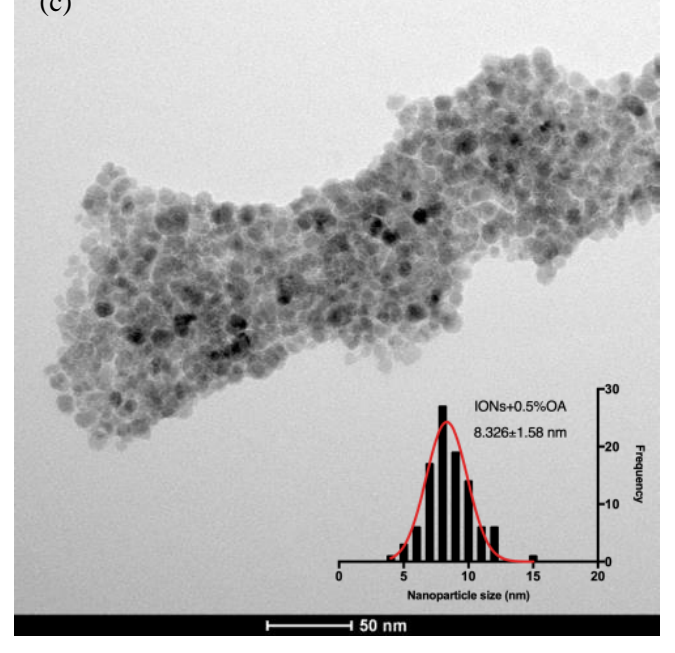

(b)

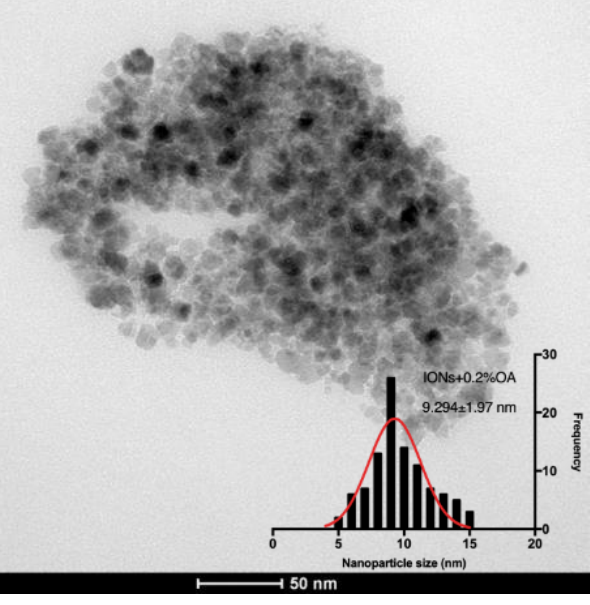

(d)

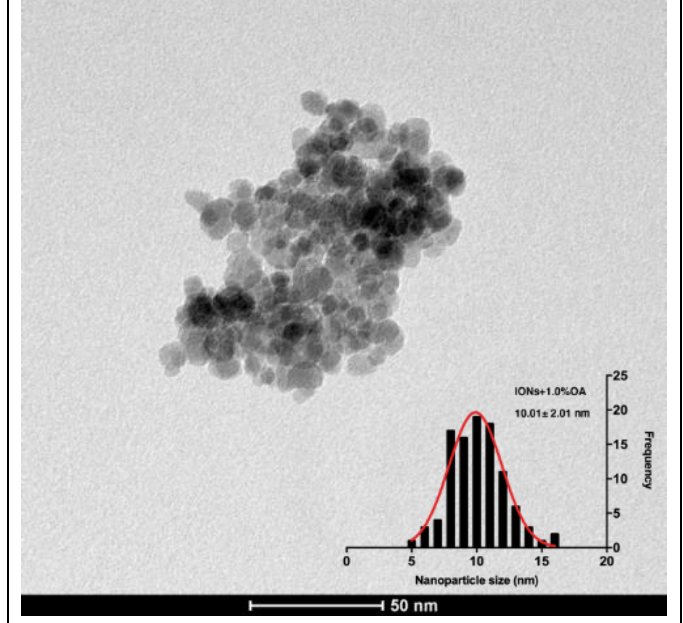

Figure 4. TEM images of a) IONs, b) IONs+0.2\%OA, v/v. c) IONs+0.5\%OA, v/v. d) IONs+1.0\%OA, v/v. with size distribution histograms.

\subsection{Dynamic light scattering (DLS) analysis}

Hydrodynamic size and size distribution of the bare and OA coated IONs were measured by DLS as shown in Fig. 5. The average diameter of the bare and 0.2, 0.5 and 1.0\%, v/v OA coated IONs were found 
as $92.1 \pm 28.1,92.5 \pm 36.2,72.3 \pm 28.5$ and $69.9 \pm 32.5 \mathrm{~nm}$, respectively. Hydrodynamic size of the IONs decreased as the OA concentration reached to $1.0 \%$ and these data revealed that OA coatings tend to decrease the agglomeration of IONs especially in the case of OA concentration higher than $0.2 \%$, v/v. As noted here and in the literature that hydrodynamic size dramatically differs from TEM identified static size, especially for nanoparticles [29]. In TEM images, the OA coating is not visible, therefore, the measured diameters are related to core iron oxide nanoparticles (IONs). Therefore, DLS gives the average hydrodynamic diameter rather than the actual diameter of nanoparticles [29, 38].
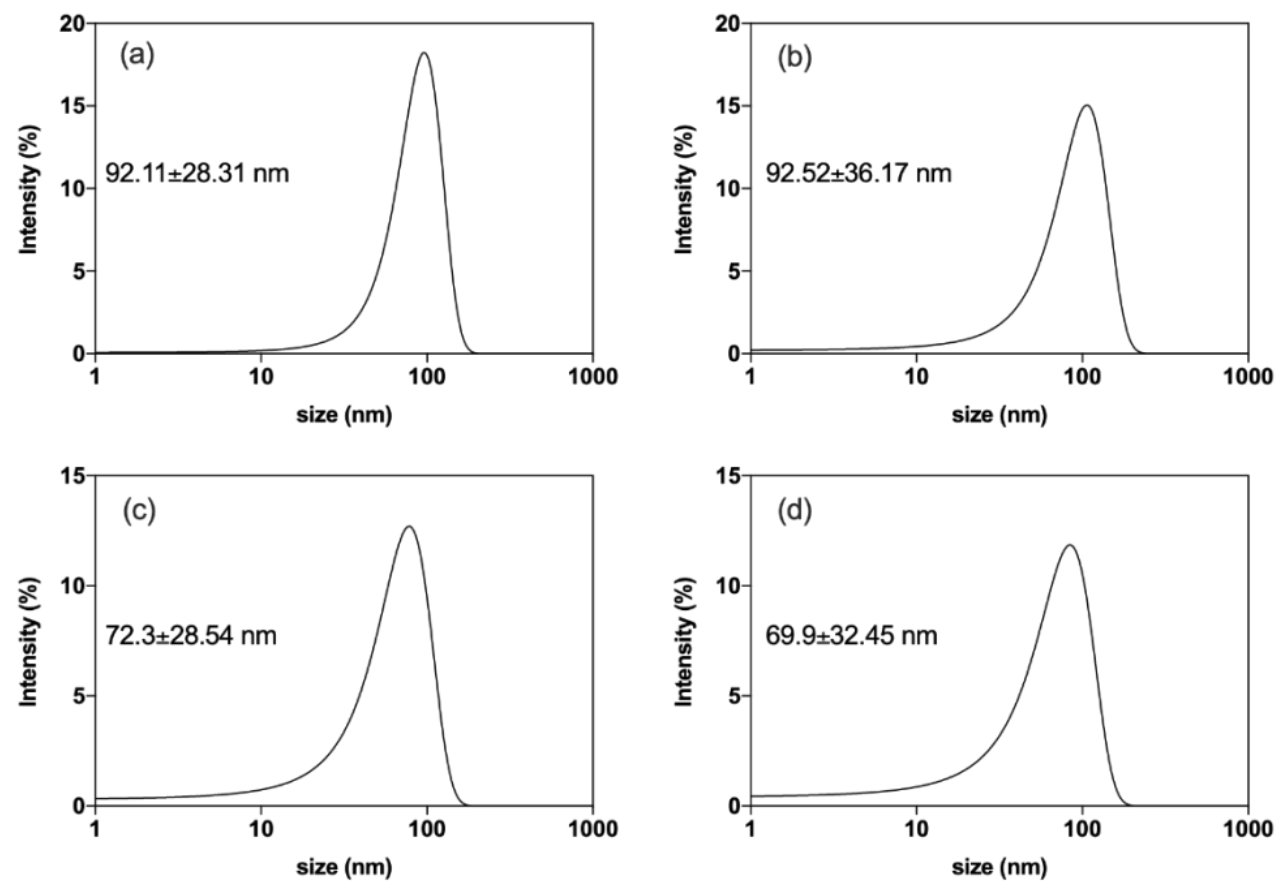

Figure 5. Particle size distribution of a) IONs, b) IONs+0.2\%OA, v/v. c) IONs+0.5\%OA, v/v. and d) IONs+1.0\%OA, v/v. based on DLS measurements. (Data were fitted with non-linear Gaussian curve fit.)

\subsection{Magnetic properties of IONs}

The magnetic properties of IONs and OA coated IONs were measured at room temperature using quantum design physical property measurement system (PPMS), as shown in Fig. 6. It is clear to find that magnetization curves of all the samples exhibit superparamagnetic behavior, in which the coercivity are close to zero. The saturation magnetization for bare and $0.2,0.5$ and $1.0 \%$, v/v OA coated IONs were 62.0, $61.5,59.5$ and $58.2 \mathrm{emu} / \mathrm{g}$, respectively, and Ms values of the OA coated IONs did not dramatically change with increasing OA concentration. The change of magnetic properties due to the OA coating present in the literature is controversial. Petcharoen et al. showed that bare magnetite (IONs), IONs+0.8\%OA, v/v and hexanoic acid coated IONs possessed 57.1, 33.3 and $58.7 \mathrm{emu} / \mathrm{g}$, Ms values, respectively. That is, the coating layer (hexanoic acid) of IONs slightly effect (1-2 emu/g) to Ms value, but Ms value for $0.8 \%$ OA coating on IONs dramatically decreased [21]. A sharp decrease on Ms values of IONs after OA coating was also reported by other studies. Liu et al. showed that the amount of OA $(2.16 \% \mathrm{v} / \mathrm{v})$ decreased the Ms value of IONs from $58 \mathrm{emu} / \mathrm{g}$ to $46 \mathrm{emu} / \mathrm{g}$ [39]. Also, Tans1k et al. observed that $4.5 \%$ (v/v) OA coating on IONs decreased Ms value from 62 to $55 \mathrm{emu} / \mathrm{g}$ [40]. On the other hand, Jovanovic et al. showed that OA coating on IONs did not affect Ms value up to $0.1 \mathrm{M}$, however, significant changes were observed at higher OA concentrations [41]. In this study, OA coating with different concentrations increased the colloidal stability of the IONs and did not affect the magnetic properties of the resultant nanoparticles. Therefore, these OA coated nanoparticles were found as good candidates for hyperthermia applications with these superior 
properties and their heating potential and SAR values were investigated via alternating current (AC) magnetic field in subsequent heating experiments.

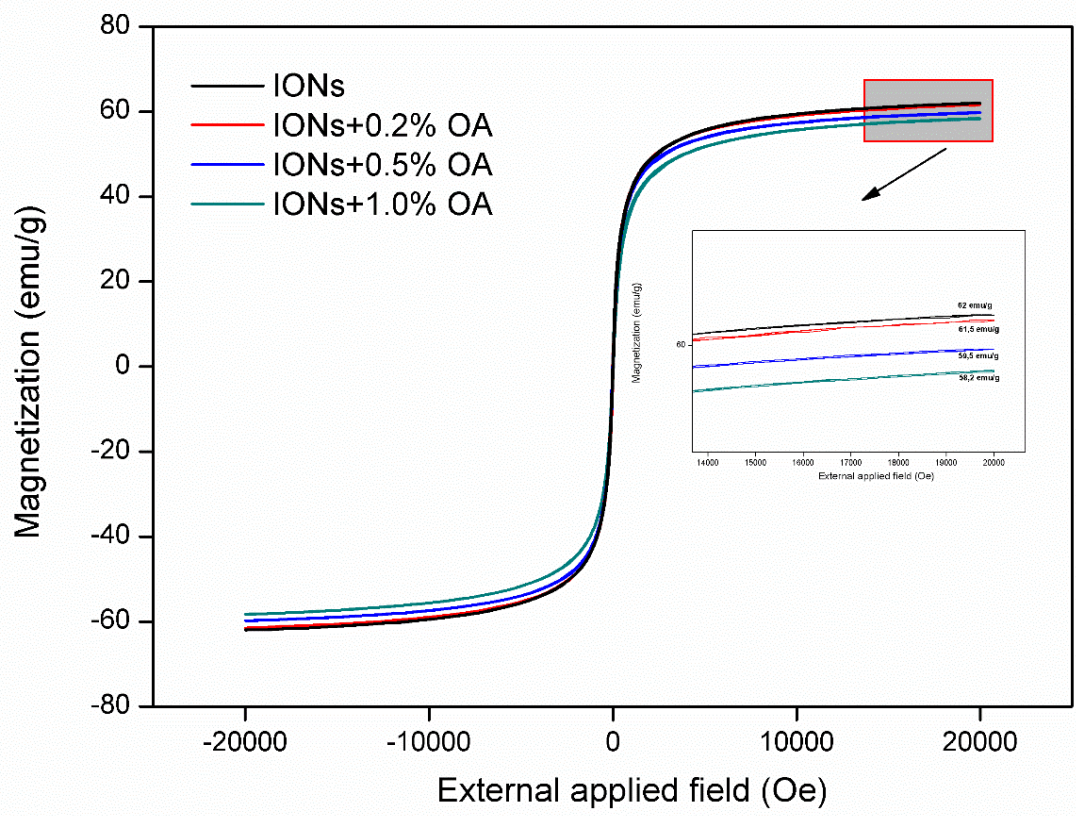

Figure 6. Magnetization $(\mathrm{M})$ versus applied magnetic field $(\mathrm{H})$ curve for IONs and IONs+OA $(0.2 \%, 0.5 \%$, $1.0 \%, \mathrm{v} / \mathrm{v})$ at room temperature. Here, $\mathrm{M}$ is expressed in terms of emu/g.

\subsection{Induction heating ability of IONs and SAR}

The induction heating capabilities of bare IONs and OA coated IONs were measured after preparing their suspension in distilled water with different IONs concentrations. Figure 7a-b represents the dynamic temperature curves for IONs and IONs+1.0\%OA, v/v under $100 \mathrm{~A}, 312 \mathrm{kHz}$ frequency of alternating magnetic field during $10 \mathrm{~min}$ for different nanoparticle concentrations (1-10 mg/mL). As shown in Fig. 7a and $\mathrm{b}, 10 \mathrm{mg} / \mathrm{mL}$ of bare IONs and IONs+1.0\%OA concentrations showed maximum temperature increase such as $26.7^{\circ} \mathrm{C}$ and $25.2{ }^{\circ} \mathrm{C}$, respectively. The increase of temperature has a linear relationship with the concentration of magnetic nanoparticles present in suspension medium. Thus, the temperature increase decreased as the nanoparticle concentrations was gradually lowered to $1 \mathrm{mg} / \mathrm{mL}$. Besides, the temperature increases for IONs+0.2\%OA, v/v and IONs+0.5\%OA, v/v $(10 \mathrm{mg} / \mathrm{mL})$ were significantly lower than bare IONs and IONs+1.0\%OA (16.4 ${ }^{\circ} \mathrm{C}$ and $19.0^{\circ} \mathrm{C}$, respectively) (Fig. 7c). In hyperthermia applications, the temperature of a tumor environment should reach the therapeutic temperature $\left(41-45^{\circ} \mathrm{C}\right)$ within few minutes [42]. In this study, the time needed for temperature to reach $45^{\circ} \mathrm{C}$ from body temperature was less than $2 \mathrm{~min}$ for IONs and IONs+1.0\%OA, v/v with concentrations higher than $5 \mathrm{mg} / \mathrm{mL}$. Due to the insufficient colloidal stability and an extra OA layer, IONs+0.2\%OA, v/v and IONs+0.5\%OA, v/v samples did not show the same heating performance as compared to bare IONs and IONs+1.0\%OA, v/v (Fig. 7d). Therefore, these findings suggest that OA coating with low concentrations was not sufficient to stabilize IONs in water indicating agglomeration and precipitation in the end. Our results revealed that IONs coated with $1.0 \% \mathrm{v} / \mathrm{v} \mathrm{OA}$, v/v showed similar heating performance with bare IONs and 1\%, v/v found as optimum OA concentration for hyperthermia applications. SAR values reflect the magnitude of heat dissipation from NPs to surrounding medium. SAR values of bare IONs and IONs+OA $(0.2 \%, 0.5 \%, 1.0 \%$, v/v) were found as $39.50,34.81,23.36$ and $45.98 \mathrm{~W} / \mathrm{g}$, respectively for nanoparticle concentrations of $10 \mathrm{mg} / \mathrm{mL}$. Ghosh et al. investigated the heating effects of $\mathrm{Fe}_{3} \mathrm{O}_{4}+\mathrm{OA}(10 \%, \mathrm{v} / \mathrm{v})$ under increasing induction heating conditions 
(100-600 A) at $265 \mathrm{kHz}$ for $10 \mathrm{~min} .100$ ampere (A) current was not sufficient for heating up to $42{ }^{\circ} \mathrm{C}$ for all tested IONs concentrations (2-20 mg) [17]. Jadhav et al. [34] prepared different concentrations of OA coated magnetic nanoparticles (MN-OA, 20\%, 10\%, 5\%, w/w) and induction heating was carried out at $400 \mathrm{~A}, 265 \mathrm{kHz}$ radiofrequency for $10 \mathrm{~min}$. The results revealed that MN-OA (10\%, w/w) showed a maximum heating up $\left(\Delta \mathrm{T} \sim 23^{\circ} \mathrm{C}\right)$. Soares et al. [16] synthesized magnetic nanoparticles coated with various $\mathrm{OA}(8 \%, 32 \%, 64 \%, 96 \%, \mathrm{w} / \mathrm{w})$ concentrations under $\mathrm{AC}$ magnetic field (24 kA m-1, $418.5 \mathrm{kHz}$ for 10 min). Induced heating ability of $8.0 \%$ of OA coating was similar to uncoated magnetic nanoparticle, whereas the heating ability of nanoparticles dramatically decreased with OA concentrations $(32 \%, 64 \%$, $96 \%, \mathrm{v} / \mathrm{v})$ increase. The results obtained in this study implied that low OA coatings on IONs can be successfully used for magnetic hyperthermia applications under relatively low frequency magnetic field.
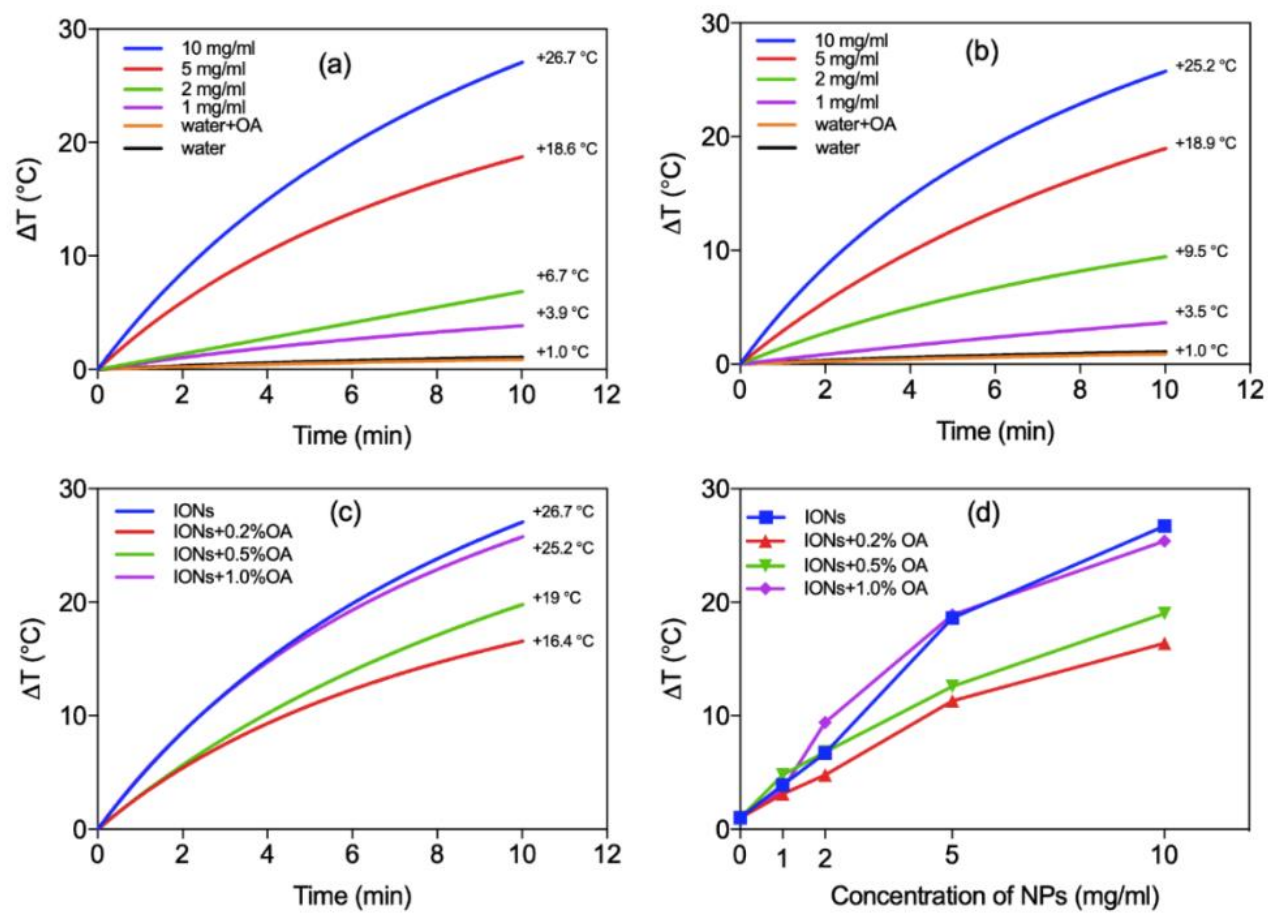

Figure 7. The time-dependent changes in the temperature of (a) IONs and (b) IONs+1.0\%OA, v/v. with different IONs concentrations, c) bare IONs and IONs with different OA coatings (IONs concentration: $10 \mathrm{mg} / \mathrm{mL}$ ) and d) The concentrationdependent changes in the temperature of all NPs (application time: $10 \mathrm{~min}$ ).

\section{CONCLUSION}

Our results revealed that capping with $1.0 \%, \mathrm{v} / \mathrm{v}$ OA enhances the hyperthermia effect of IONs dramatically compared to $0.2 \% \mathrm{OA}$, v/v and $0.5 \% \mathrm{OA}, \mathrm{v} / \mathrm{v}$. The enhanced hyperthermia effect of IONs+1.0\%OA, v/v was attributed to their high dispersion performance without formation of large agglomerates in water. IONs coated with low OA concentration can be successfully used in magnetic nano hyperthermia applications. As a result, the heating effect highly depends on physical and chemical properties of IONs, hyperthermia exposure systems, measurement conditions, nanoparticle size distributions and especially colloidal stability of IONs. 
This work was supported by the Scientific and Technological Research and Technological Research Council of Turkey (TUBITAK), Grant no: SBAG-118S027. We are grateful to Dr. Mehmet Burak Kaynar for his help to the XRD, VSM and hyperthermia measurements.

\section{REFERENCES}

[1] J. Beik et al., "Nanotechnology in hyperthermia cancer therapy: From fundamental principles to advanced applications," Journal of controlled release : official journal of the Controlled Release Society, vol. 235, pp. 205-21, Aug 10 2016, doi: 10.1016/j.jconrel.2016.05.062.

[2] H. Liu et al., "Application of iron oxide nanoparticles in glioma imaging and therapy: from bench to bedside," Nanoscale, vol. 8, no. 15, pp. 7808-7826, 2016.

[3] K. El-Boubbou, "Magnetic iron oxide nanoparticles as drug carriers: Preparation, conjugation and delivery," Nanomedicine, vol. 13, no. 8, pp. 929-952, 2018.

[4] R. Wildeboer, P. Southern, and Q. Pankhurst, "On the reliable measurement of specific absorption rates and intrinsic loss parameters in magnetic hyperthermia materials," Journal of Physics D: Applied Physics, vol. 47, no. 49, p. 495003, 2014.

[5] H. A. Albarqi et al., "Biocompatible Nanoclusters with High Heating Efficiency for Systemically Delivered Magnetic Hyperthermia," ACS Nano, vol. 13, no. 6, pp. 6383-6395, Jun 25 2019, doi: 10.1021/acsnano.8b06542.

[6] A. E. Deatsch and B. A. Evans, "Heating efficiency in magnetic nanoparticle hyperthermia," Journal of Magnetism and Magnetic Materials, vol. 354, pp. 163-172, 2014, doi: 10.1016/j.jmmm.2013.11.006.

[7] W. Xie et al., "Shape-, size-and structurecontrolled synthesis and biocompatibility of iron oxide nanoparticles for magnetic theranostics," Theranostics, vol. 8, no. 12, p. 3284, 2018.
[8] M. Cano et al., "Partial PEGylation of superparamagnetic iron oxide nanoparticles thinly coated with amine-silane as a source of ultrastable tunable nanosystems for biomedical applications," Nanoscale, vol. 9, no. 2, pp. 812-822, Jan 5 2017, doi: 10.1039/c6nr07462f.

[9] M. Liu et al., "Synthesis of magnetic molecularly imprinted polymers for the selective separation and determination of metronidazole in cosmetic samples," Anal Bioanal Chem, vol. 407, no. 13 , pp. 3875-80, May 2015, doi: 10.1007/s00216-015-8592-7.

[10] F. Shubitidze, K. Kekalo, R. Stigliano, and I. Baker, "Magnetic nanoparticles with high specific absorption rate of electromagnetic energy at low field strength for hyperthermia therapy," J Appl Phys, vol. 117, no. 9, p. 094302, Mar 7 2015, doi: 10.1063/1.4907915.

[11] L. Xie, W. Jin, H. Chen, and Q. Zhang, "Superparamagnetic Iron Oxide Nanoparticles for Cancer Diagnosis and Therapy," J Biomed Nanotechnol, vol. 15, no. 2, pp. 215-416, Feb 1 2019, doi: 10.1166/jbn.2019.2678.

[12] J. Cai, Y. Q. Miao, B. Z. Yu, P. Ma, L. Li, and H. M. Fan, "Large-Scale, Facile Transfer of Oleic Acid-Stabilized Iron Oxide Nanoparticles to the Aqueous Phase for Biological Applications," Langmuir, vol. 33, no. 7, pp. 1662-1669, Feb 21 2017, doi: 10.1021/acs.langmuir.6b03360.

[13] L. H. Reddy, J. L. Arias, J. Nicolas, and P. Couvreur, "Magnetic nanoparticles: design and characterization, toxicity and biocompatibility, pharmaceutical and biomedical applications," Chemical reviews, vol. 112, no. 11, pp. 5818-5878, 2012. 
[14] R. Gupta et al., "Effect of Oleic Acid Coating of Iron Oxide Nanoparticles on Properties of Magnetic Polyamide-6 Nanocomposite," JOM, vol. 71, no. 9, pp. 3119-3128, 2019.

[15] C. W. Lai, F. W. Low, M. F. Tai, and S. B. Abdul Hamid, "Iron oxide nanoparticles decorated oleic acid for high colloidal stability," Advances in Polymer Technology, vol. 37, no. 6, pp. 1712-1721, 2018.

[16] P. I. Soares et al., "Iron oxide nanoparticles stabilized with a bilayer of oleic acid for magnetic hyperthermia and MRI applications," Applied Surface Science, vol. 383, pp. 240-247, 2016.

[17] R. Ghosh et al., "Induction heating studies of $\mathrm{Fe} 3 \mathrm{O} 4$ magnetic nanoparticles capped with oleic acid and polyethylene glycol for hyperthermia," Journal of Materials Chemistry, vol. 21, no. 35, 2011, doi: 10.1039/c1jm10092k.

[18] R. Araújo-Neto et al., "Monodisperse sodium oleate coated magnetite high susceptibility nanoparticles for hyperthermia applications," J Magn Magn Mater, vol. 364, pp. 72-79, 2014.

[19] S. Sánchez-Cabezas, R. Montes-Robles, J. Gallo, F. Sancenón, and R. Martínez-Máñez, "Combining magnetic hyperthermia and dual $\mathrm{T} 1 / \mathrm{T}$ 2 MR imaging using highly versatile iron oxide nanoparticles," Dalton Transactions, vol. 48, no. 12, pp. 3883-3892, 2019.

[20] R. Massart, "Preparation of aqueous magnetic liquids in alkaline and acidic media," IEEE transactions on magnetics, vol. 17, no. 2, pp. 1247-1248, 1981.

[21] K. Petcharoen and A. Sirivat, "Synthesis and characterization of magnetite nanoparticles via the chemical co-precipitation method," Materials Science and Engineering: B, vol. 177, no. 5, pp. 421-427, 2012, doi: 10.1016/j.mseb.2012.01.003.

[22] C. Iacovita et al., "Small versus large iron oxide magnetic nanoparticles: hyperthermia and cell uptake properties," Molecules, vol. 21, no. 10, p. 1357, 2016.
[23] I. Hilger, K. Frühauf, W. Andrä, R. Hiergeist, R. Hergt, and W. A. Kaiser, "Heating potential of iron oxides for therapeutic purposes in interventional radiology," Academic radiology, vol. 9, no. 2, pp. 198-202, 2002.

[24] F. Wang et al., "Synthesis and characterization of superparamagnetic $\mathrm{Fe} 3 \mathrm{O} 4$ nanoparticles modified with oleic acid," Integrated Ferroelectrics, vol. 153, no. 1, pp. 92-101, 2014.

[25] Q. Zhang, C. Wang, L. Qiao, H. Yan, and K. Liu, "Superparamagnetic iron oxide nanoparticles coated with a folate-conjugated polymer," Journal of Materials Chemistry, vol. 19, no. 44, pp. 83938402, 2009.

[26] P. I. Soares et al., "Effects of surfactants on the magnetic properties of iron oxide colloids," J Colloid Interface Sci, vol. 419, pp. 46-51, Apr 1 2014, doi: 10.1016/j.jcis.2013.12.045.

[27] I. O. Wulandari, H. Sulistyarti, A. Safitri, D. J. H. Santjojo, and A. Sabarudin, "Development of synthesis method of magnetic nanoparticles modified by oleic acid and chitosan as a candidate for drug delivery agent," Journal of Applied Pharmaceutical Science, vol. 9, no. 07, pp. 001-011, 2019.

[28] K. S. Sharma et al., "Synthesis and characterization of monodispersed water dispersible $\mathrm{Fe} 3 \mathrm{O} 4$ nanoparticles and in vitro studies on human breast carcinoma cell line under hyperthermia condition," Sci Rep, vol. 8, no. 1, p. 14766, Oct 3 2018, doi: 10.1038/s41598-01832934-w.

[29] J. Ibarra et al., "Synthesis and characterization of magnetite/PLGA/chitosan nanoparticles," Materials Research Express, vol. 2, no. 9, 2015, doi: 10.1088/2053-1591/2/9/095010.

[30] L. Zhang, R. He, and H.-C. Gu, "Oleic acid coating on the monodisperse magnetite nanoparticles," Applied Surface Science, vol. 253, no. 5, pp. 2611-2617, 2006, doi: 10.1016/j.apsusc.2006.05.023. 
[31] N. Wu, L. Fu, M. Su, M. Aslam, K. C. Wong, and V. P. Dravid, "Interaction of fatty acid monolayers with cobalt nanoparticles," Nano letters, vol. 4, no. 2, pp. 383-386, 2004.

[32] K. Yang, H. Peng, Y. Wen, and N. Li, "Reexamination of characteristic FTIR spectrum of secondary layer in bilayer oleic acid-coated Fe3O4 nanoparticles," Applied Surface Science, vol. 256, no. $10, \quad$ pp. 3093-3097, 2010, doi: 10.1016/j.apsusc.2009.11.079.

[33] L. N. Okassa et al., "Optimization of iron oxide nanoparticles encapsulation within poly $(\mathrm{d}, 1-$ lactide-co-glycolide) sub-micron particles," Eur J Pharm Biopharm, vol. 67, no. 1, pp. 31-8, Aug 2007, doi: 10.1016/j.ejpb.2006.12.020.

[34] N. V. Jadhav et al., "Synthesis of oleic acid functionalized $\mathrm{Fe} 3 \mathrm{O} 4$ magnetic nanoparticles and studying their interaction with tumor cells for potential hyperthermia applications," Colloids Surf B Biointerfaces, vol. 108, pp. 158-68, Aug 12013 , doi: 10.1016/j.colsurfb.2013.02.035.

[35] G. Sharma and P. Jeevanandam, "Synthesis of self-assembled prismatic iron oxide nanoparticles by a novel thermal decomposition route," RSC Adv., vol. 3, no. 1, pp. 189-200, 2013, doi: $10.1039 / \mathrm{c} 2 \mathrm{ra} 22004 \mathrm{k}$.

[36] Z. Shan, W.-S. Yang, X. Zhang, Q.-M. Huang, and H. Ye, "Preparation and characterization of carboxyl-group functionalized superparamagnetic nanoparticles and the potential for bio-applications," Journal of the Brazilian Chemical Society, vol. 18, no. 7, pp. 1329-1335, 2007.
[37] M. Mahdavi et al., "Synthesis, surface modification and characterisation of biocompatible magnetic iron oxide nanoparticles for biomedical applications," Molecules, vol. 18, no. 7, pp. 753348, Jun 27 2013, doi: 10.3390/molecules18077533.

[38] S. Prabha, W.-Z. Zhou, J. Panyam, and V. Labhasetwar, "Size-dependency of nanoparticlemediated gene transfection: studies with fractionated nanoparticles," Int J Pharmaceut, vol. 244, no. 1-2, pp. 105-115, 2002.

[39] X. Liu, M. D. Kaminski, Y. Guan, H. Chen, H. Liu, and A. J. Rosengart, "Preparation and characterization of hydrophobic superparamagnetic magnetite gel," Journal of magnetism and magnetic materials, vol. 306, no. 2, pp. 248-253, 2006.

[40] G. Tansık, A. Yakar, and U. Gündüz, "Tailoring magnetic PLGA nanoparticles suitable for doxorubicin delivery," Journal of nanoparticle research, vol. 16, no. 1, p. 2171, 2014.

[41] S. Jovanović, M. Spreitzer, M. Tramšek, Z. Trontelj, and D. Suvorov, "Effect of oleic acid concentration on the physicochemical properties of cobalt ferrite nanoparticles," The Journal of Physical Chemistry C, vol. 118, no. 25, pp. 1384413856, 2014.

[42] T.-C. Lin, F.-H. Lin, and J.-C. Lin, "In vitro feasibility study of the use of a magnetic electrospun chitosan nanofiber composite for hyperthermia treatment of tumor cells," Acta biomaterialia, vol. 8, no. 7, pp. 2704-2711, 2012. 
Natural \& Applied Sciences Journal Vol. 2 (2) 201930 